\title{
Observation of Spin Hall Effect in Photon Tunneling via Weak Measurements
}

SUBJECT AREAS:

OPTICAL PHYSICS

PHOTONIC DEVICES

Received

11 September 2014

Accepted

20 November 2014

Published

9 December 2014

Correspondence and requests for materials should be addressed to H.L. (hailuluo@hnu. edu.cn)

\author{
Xinxing Zhou', Xiaohui Ling², Zhiyou Zhang' ${ }^{2}$, Hailu Luo' \& Shuangchun Wen'
}

'Laboratory for Spin Photonics, School of Physics and Electronics, Hunan University, Changsha 410082, China, ${ }^{2}$ SZU-NUS Collaborative Innovation Center for Optoelectronic Science \& Technology, Shenzhen University, Shenzhen 51 8060, China.

Photonic spin Hall effect (SHE) manifesting itself as spin-dependent splitting escapes detection in previous photon tunneling experiments due to the fact that the induced beam centroid shift is restricted to a fraction of wavelength. In this work, we report on the first observation of this tiny effect in photon tunneling via weak measurements based on preselection and postselection technique on the spin states. We find that the spin-dependent splitting is even larger than the potential barrier thickness when spin-polarized photons tunneling through a potential barrier. This photonic SHE is attributed to spin-redirection Berry phase which can be described as a consequence of the spin-orbit coupling. These findings provide new insight into photon tunneling effect and thereby offer the possibility of developing spin-based nanophotonic applications.

$\mathrm{T}$ he tunneling effect is one of the important cornerstones of the quantum mechanics ${ }^{1}$, where particle such as electron may penetrate through an impenetrable potential barrier. Photon also has the possibility to penetrate through classically impenetrable potential barrier, which is called the photon tunneling ${ }^{2-4}$. In particular, frustrated total internal reflection is considered as a classical analogy of quantum-mechanical tunneling. In this case, photons play the role of electrons and can penetrate across the air gap between two right angle prisms when the incident angle beyonds the critical angle $\mathrm{e}^{5-8}$. It is well known that photon has an additional degree of freedom of spin, the photonic spin Hall effect (SHE) manifesting as the spin-dependent splitting of left- and righthanded circularly polarized components can occur in photon tunneling due to spin-orbit coupling. In fact, the photonic SHE is sometimes referred to as the Imbert-Fedorov phenomenon which was theoretically proposed by Fedorov and experimentally verified by Imbert ${ }^{9,10}$. For optical wavelengths, the photonic SHE is very weak and the corresponding spin-dependent splitting is restricted to a few tens of nanometers. It is the possible reason for this tiny effect escaping detection in previous photon tunneling experiments.

The weak measurements based on preselection and postselection states is a promising method for measuring tiny perturbations of the quantum system ${ }^{11-15}$. An observable of a system is firstly coupled to a separate degree of freedom (known as the meter corresponding to an ancillary measuring device), and then the information about the state of observable is read out from the meter, which leaves the state almost undisturbed. Obtaining the desired output involves: initializing a quantum system of interesting state standing for the preselection, the weak coupling between observable and meter through the system operator $\hat{A}$, and the postselection with definite final state. If we select the suitable preselection and postselection states in quantum system, the distinguished expectation values (eigenvalues) can be obtained in the meter. Until now the weak measurements have been used to resolve some important issues such as ultrasensitive beam deflection measurement ${ }^{16}$, measurement of small longitudinal phase shifts $^{17}$, direct measurement of the quantum wavefunction ${ }^{18}$, observation of the average trajectories of single photons ${ }^{19}$, and full characterization of polarization states of light ${ }^{20}$.

In this work, we report on the first observation of photonic SHE in tunneling via weak measurements. A simple and typical $A B A$ structure is considered as the tunneling model. $A$ stands for the right angle prism and $B$ is the metal tunneling potential barrier allowing for only evanescent waves. We show that the photonic SHE in tunneling manifesting itself as the spin-dependent splitting due to the spin-orbit coupling. With suitable preselecion and postselection technique on the spin states, the transverse shifts of spin (polarization) components can be measured with desired accuracy. Our findings offer a route for spin-controlled nanophotonic applications and can be extrapolated to other physical systems.

\section{Results}

Theoretical analysis. Figure 1 illustrates the SHE of photons tunneling through a three-layer barrier structure. A metal film $(\mathrm{Au})$ acting as a tunneling barrier is bounded by two identical semi-infinite medium (two right angle 


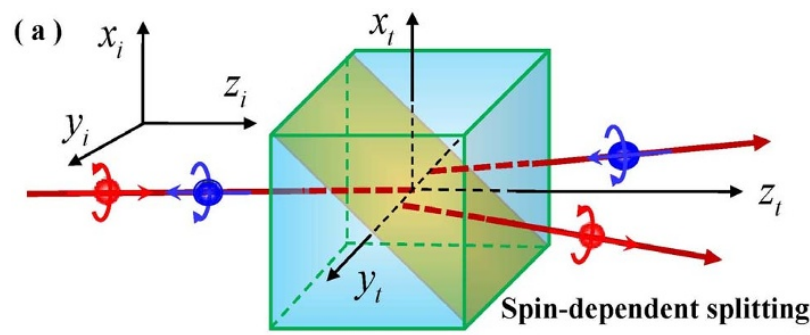

( b )

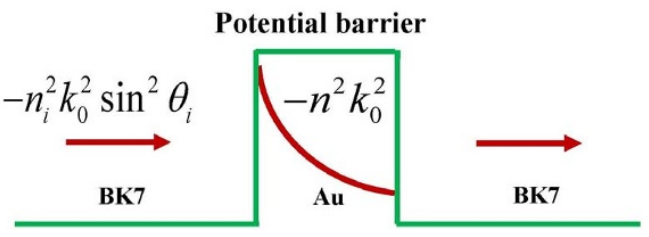

Figure 1 $\mid$ (a) Schematic of the photonic SHE phenomenon in tunneling. A linearly polarized beam transmits through the potential barrier structure composed of two BK7 prisms embed with an Au film and then splits into left- and right-handed circularly polarized components, respectively. Here, the incident angle $\theta_{i}$ is fixed to $45^{\circ}$ (at the interface of metal film). (b) shows the potential diagram of photon tunneling. The total energy of the incident photon is $-k_{x}^{2}=-n_{i}^{2} k_{0}^{2} \sin ^{2} \theta_{i}$. The central metal core is the tunnel barrier and its potential can be described as $-n^{2} k_{0}^{2}$.

BK7 prisms) on either side and only permits evanescent wave transmission. When the photons tunnel through the barrier, the photonic SHE occurs and the transmitted beam splits by a fraction of wavelength into its two left- and right-handed circularly polarized components. Here, the incident angle $\theta_{i}$ is fixed to $45^{\circ}$. The photonic SHE is the photonic version of the SHE in electronic systems, in which the photons play the role of the spin charges, and a refractive index gradient plays the role of the electric potential gradient $^{21-23}$.

An equivalent potential for photons, analogy to quantum mechanics, can be introduced. The equivalent parameters to the potential and total incident energy are $-n^{2} k_{0}^{2}$ and $-k_{x}^{2}=-n_{i}^{2} k_{0}^{2} \sin ^{2} \theta_{i}$, respectively ${ }^{4}$. Here, $n$ is the refractive index of potential barrier, $n_{i}$ stands for the refractive index of incident medium, and $k_{0}$ is the wave number in free space. Generally, the ordinary dielectrics own negative potential. The dielectric can be regarded as a tunneling barrier when the energy of the incident photon is below the potential of this barrier. Therefore it needs to modulate the incident angle so that the energy of the incident photon can be reduced sufficiently. Under this tunneling condition the light beam undergoes total internal reflection. Materials with a purely imaginary refractive index (ideal metals) can be seen as tunneling barriers for all angles of incidence because its potential is larger than zero. In this case, we note that the metal film can act as a tunneling barrier for all angles of incidence because the real part of its refractive index is far less than the imaginary part ${ }^{4}$.

We can describe the photonic SHE as the result of spin-orbit coupling. For a paraxial beam, the incident polarization states are chosen as horizontal $|H\rangle$ or vertical $|V\rangle$. In the spin basis, the horizontal and vertical polarization states can be expressed as $|H\rangle=(|+\rangle+|-\rangle) / \sqrt{2}$ and $|V\rangle=(|+\rangle-i|-\rangle) / \sqrt{2}$. Owing to the transverse nature of the photon polarization, the polarizations associated with the corresponding plane-wave components undergo different rotations ${ }^{23}$. When the photons tunnel through the barrier, the evolution of the states is described as $\left|k_{y}\right\rangle|H\rangle \rightarrow\left|k_{y}\right\rangle(|H\rangle+$ $\left.k_{y} \delta^{H}|V\rangle\right)$ and $\left|k_{y}\right\rangle|V\rangle \rightarrow\left|k_{y}\right\rangle\left(|V\rangle+k_{y} \delta^{V}|H\rangle\right)$. In the spin basis, the states of tunneling field can be obtained:

$$
\begin{gathered}
|H\rangle \rightarrow \frac{t_{p}}{\sqrt{2}}\left[\exp \left(-i k_{y} \delta^{H}\right)|+\rangle+\exp \left(+i k_{y} \delta^{H}\right)|-\rangle\right], \\
|V\rangle \rightarrow \frac{i t_{s}}{\sqrt{2}}\left[-\exp \left(-i k_{y} \delta^{V}\right)|+\rangle+\exp \left(+i k_{y} \delta^{V}\right)|-\rangle\right] .
\end{gathered}
$$

In the above equations, $\delta^{H}=\left(1-t_{s} / t_{p}\right) \cot \theta_{i} / k_{0}, \delta^{V}=\left(1-t_{p} / t_{s}\right) \cot \theta_{i} /$ $k_{0}$. This photonic SHE is attributed to spin-redirection Berry phase $\exp \left(-i \hat{\sigma}_{3} k_{y} \delta^{H, V}\right)$ which is corresponding to the spin-orbit coupling.

Because a layered metal film nanostructure is introduced as the tunneling model, we need to know the generalized Fresnel transmission coefficients of the barrier by calculating the explicit solution of the boundary conditions at the either interface

$$
t_{p, s}=\frac{T_{p, s} T_{p, s}^{\prime} \exp \left(i k_{0} \sqrt{n^{2}-\sin ^{2} \theta_{i} d}\right)}{1+T_{p, s} T_{p, s}^{\prime} \exp \left(2 i k_{0} \sqrt{n^{2}-\sin ^{2} \theta_{i} d}\right)} .
$$

Here, $T_{p, s}$ and $T_{p, s}^{\prime}$ are the Fresnel transmission coefficients at the first interface and second interface of the tunneling barrier, respectively. $d$ represents the thickness of the tunneling barrier. In this work, the photonic SHE manifests as the spin-dependent splitting of two spin components in position space. We note that another type of phenomenon manifesting as spin-dependent splitting in momentum space called optical Rashba effect has been observed in a plasmoninc metasurface due to its inversion asymmetric structure geometry ${ }^{24}$. Unlike here, the underlying mechanism is attributed to the geometric Pancharatnam Berry phase.

In the following, we calculate the shifts of these two spin components. The wavefunction of tunneling photons is composed of the packet spatial extent $\phi\left(k_{y}\right)$ and the polarization description $|H, V\rangle$ :

$$
\left|\Phi^{H, V}\right\rangle=\int d k_{y} \phi\left(k_{y}\right)\left|k_{y}\right\rangle|H, V\rangle
$$

After photons tunneling through the barrier, the initial state $\left|\Phi_{\text {inital }}^{H, V}\right\rangle$ evolves into the final state $\left|\Phi_{\text {final }}^{H, V}\right\rangle$. As a result of spin-orbit coupling, the displacements of the two spin components compared to the geometrical-optics prediction are given by

$$
\delta_{| \pm\rangle}^{H, V}=\frac{\left\langle\Phi^{H, V}\left|i \partial_{\mathbf{k}_{\perp}}\right| \Phi^{H, V}\right\rangle}{\left\langle\Phi^{H, V} \mid \Phi^{H, V}\right\rangle} .
$$

We suppose $\phi\left(k_{y}\right)$ is a Gaussian wave function, the transverse displacements can be obtained as

$$
\begin{aligned}
& \delta_{| \pm\rangle}^{H}= \pm \frac{\lambda}{2 \pi}\left[1-\frac{\left|t_{s}\right|}{\left|t_{p}\right|} \cos \left(\varphi_{s}-\varphi_{p}\right)\right] \cot \theta_{i}, \\
& \delta_{| \pm\rangle}^{V}= \pm \frac{\lambda}{2 \pi}\left[1-\frac{\left|t_{p}\right|}{\left|t_{s}\right|} \cos \left(\varphi_{p}-\varphi_{s}\right)\right] \cot \theta_{i},
\end{aligned}
$$

where $t_{p, s}=\left|t_{p, s}\right| \exp \left(i \varphi_{p, s}\right)$ and $\lambda$ is wavelength of the incident beam.

For optical wavelength, the spin-dependent splitting is restricted to a few tens of nanometers (See supplementary materials for initial shifts of photonic SHE in tunneling), and therefore the actual equipment can not distinguish it directly. We resolve this problem by using the precise signal enhancement technique called quantum weak measurements proposed by Aharonov et al. ${ }^{11}$. The weak measurements of photonic SHE in tunneling can be done through the following steps (weak value amplification). Firstly, the system is prepared with a fixed initial state $\left|\psi_{1}\right\rangle$, which is called the preselection. And then, with the weak interaction, the observable $\hat{A}$ (with eigenstates $|+\rangle$ and $|-\rangle)$ is coupled to the transverse spatial distribution (the meter) of the Gaussian wave function with the Hamiltonian interaction $H_{I}=$ $k_{y} \hat{\mathbf{A}} \delta^{H, V}$. Here, the $\delta^{H, V}$ (spin shifts) also stand for the weak coupling strength that we want to estimate. However, the spin-dependent 

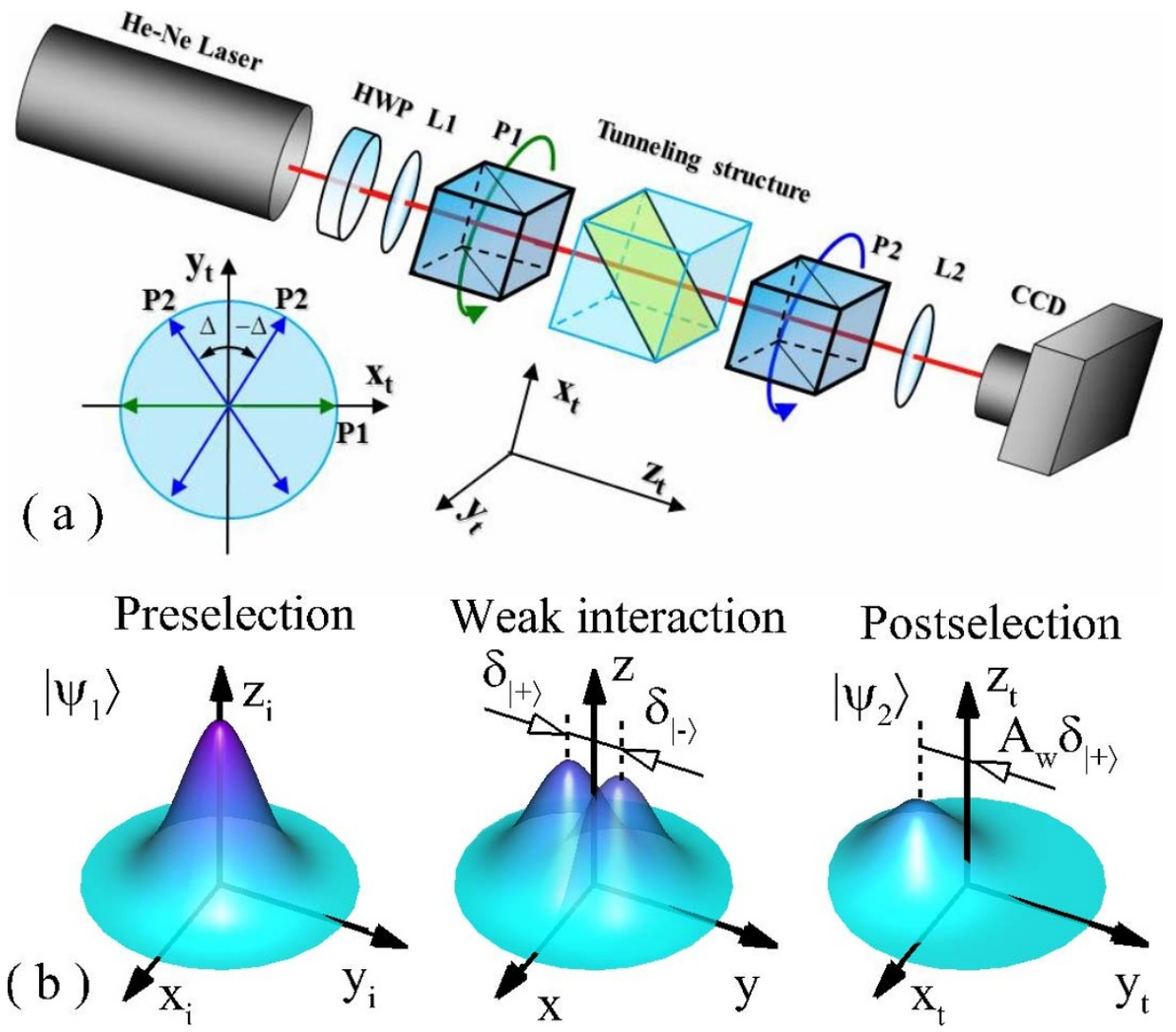

Figure 2 (a) Experimental setup: The tunneling potential barrier structure is composed of two right angle BK7 prisms (refractive index $n=1.515$ at $632.8 \mathrm{~mm}$ ) embed with Au film. HWP, half-wave plate (for adjusting the intensity). L1 and L2, lenses with effective focal length $50 \mathrm{~mm}$ and $250 \mathrm{~mm}$, respectively. P1 and P2, Glan Laser polarizers. CCD, charge-coupled device (Coherent LaserCam-HR). The light source is a $21 \mathrm{~mW}$ linearly polarized HeNe laser at $632.8 \mathrm{~nm}$ (Thorlabs HNL210L-EC). The inset describes the preselection and postselection with P1 and P2, respectively. (b) A weak measurement with preselection and postselection. System is preselected in state $\left|\psi_{1}\right\rangle$. The weak interaction correlates the meter with the eigenstates of the measured observable $\hat{\mathbf{A}}$. A postselection on the system in state $\left|\psi_{2}\right\rangle$ gives rise to an interference in the meter, shifting it to its final position proportional to $A_{w}$.

displacements generated by the photonic SHE are much smaller than the width of the transverse distribution resulting in the meter states with different spin eigenstates overlap to a large extent. Finally, with the suitable postselection state $\left|\psi_{2}\right\rangle$, an enhanced shift in the meter distribution can be obtained.

In the above weak value amplification process, the preselection and postselection states $\left|\psi_{1}\right\rangle$ and $\left|\psi_{2}\right\rangle$ determine the so-called weak value $A_{w}$ of the photon helicity:

$$
A_{w}=\frac{\left\langle\psi_{2}|\hat{\mathbf{A}}| \psi_{1}\right\rangle}{\left\langle\psi_{2} \mid \psi_{1}\right\rangle} .
$$

Here, the mean pointer position of the meter will experience a shift proportional to the weak value. By choosing a nearly orthogonal pair of preselection and postselection states, we can obtain a large weak value corresponding to an amplified signal output, which would give us a chance to estimate the coupling parameter with high accuracy. Generally, the weak value is a complex number and is not constrained to the eigenvalue range for the observable. The weak value amplification has many technical merits and can effectively suppress the technical noise ${ }^{25,26}$. We note that the imaginary weak value also corresponds to a displacement of the meter in momentum space (the mean pointer momentum), which obtains the possibility of even larger amplification following the beam free evolution ${ }^{23}$. This process can be seen as propagation amplification that produces the amplified factor $F$ which depends on the initial state and its free evolution before detection. Therefore, we can get the final pointer shift of meter proportional to the modified weak value $A_{w}^{\text {mod }}=F\left|A_{w}\right|$.
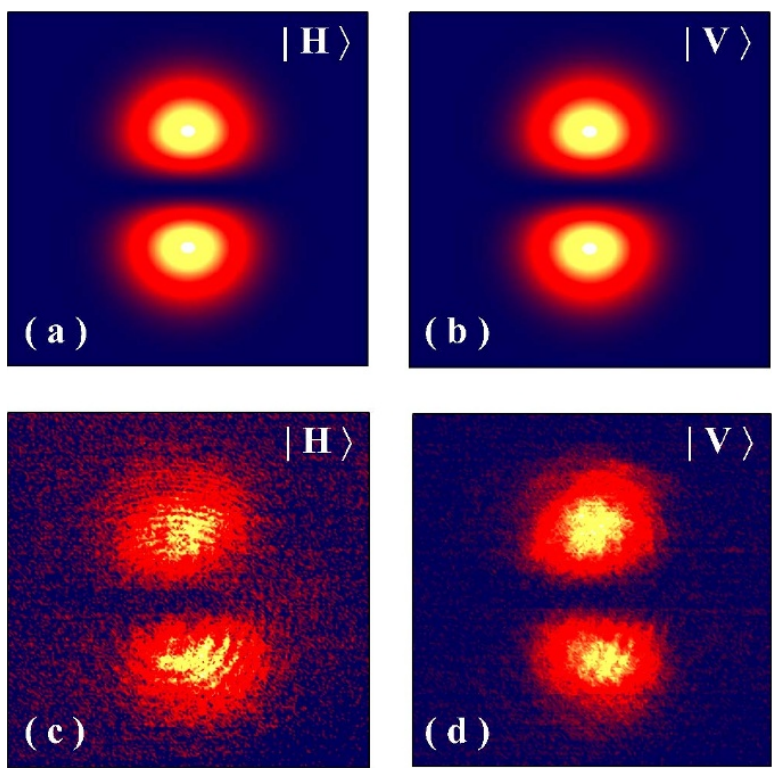

Figure 3 Intensity of the cross-polarized components of the beam tunneling through the barrier when the preselection and postselection states are orthogonal. The initial states are chosen as horizontal (left column) and vertical polarizations (right column). [(a), (b)] Theoretical results; [(c), (d)] Experimental results. The incident angle is fixed to $45^{\circ}$ and the potential barrier thickness is $12 \mathrm{~nm}$. 

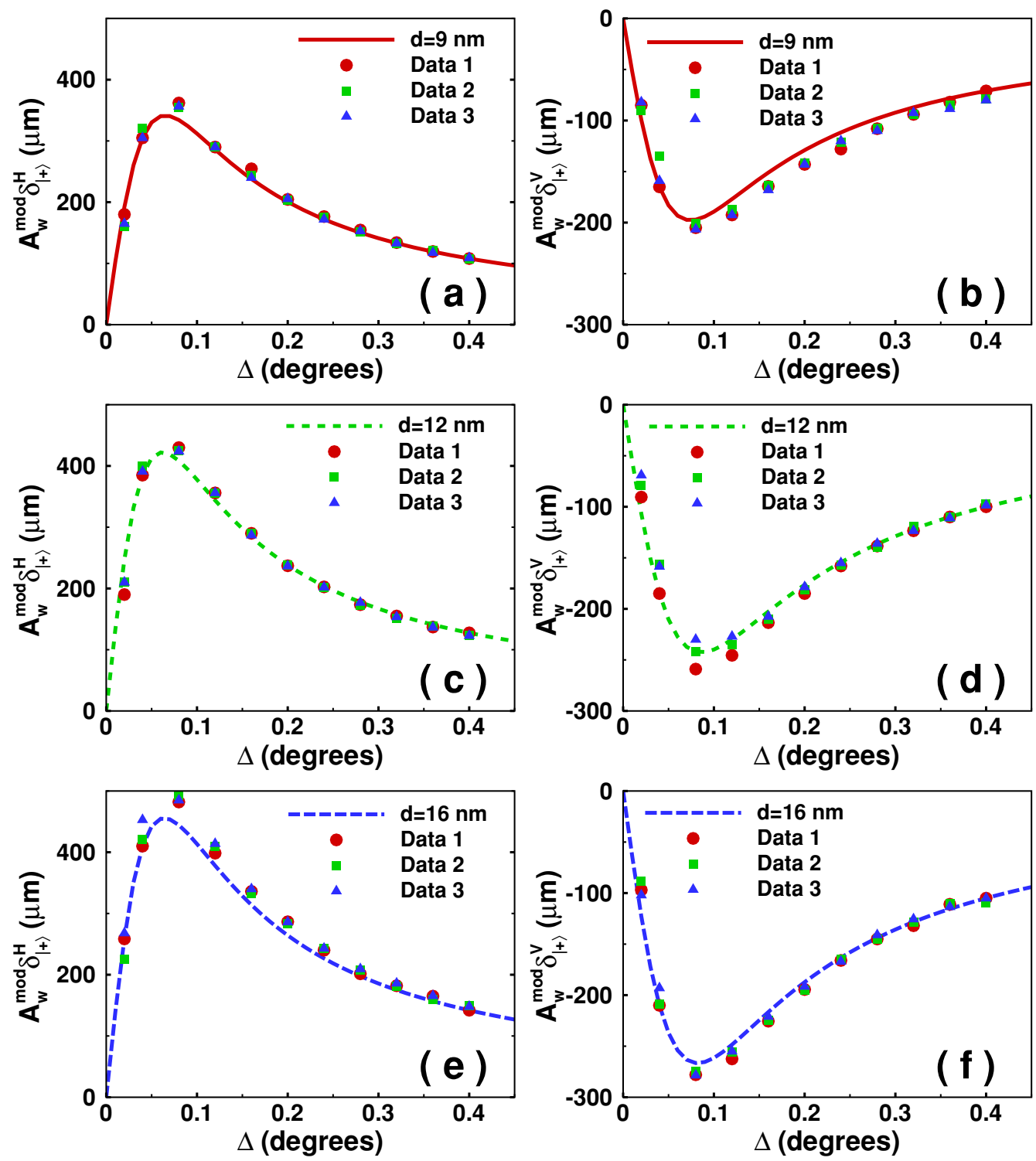

Figure $4 \mid$ The amplified shifts of spin-dependent splitting in photon tunneling under the condition of horizontal (left column) and vertical polarizations (right column) with different potential barrier thicknesses: [(a), (b)] $9 \mathrm{~nm},[(\mathrm{c}),(\mathrm{d})] 12 \mathrm{~nm}$, and [(e), (f)] $16 \mathrm{~nm}$. $A_{w}^{\text {mod }}$ represents the modified weak value of the weak measurements. The lines indicate the theoretical value and the circle, square, and triangle show the experimental data for three different areas of the tunneling sample (the error ranges are less than $10 \mu \mathrm{m}$ ).

Experimental results. Our experimental setup is schematically shown in Fig. 2(a). We construct a three-layer structure which consists of an Au film and two $45^{\circ} \mathrm{BK} 7$ right angle prisms. When photons enter the potential barrier structure, the photonic SHE happens allowing for the spin-dependent splitting in the transverse direction. The amplified effect can be obtained through the preselection and postselection [Fig. 2(b)]. A Gauss beam generated by a He-Ne laser is firstly focused by a short-focal-length lens (L1). Then, we use the polarizer P1 to get the preselection state $\left|\psi_{1}\right\rangle=|H\rangle$ or $|V\rangle$. Finally, the beam passes through the second polarizer P2 to obtain the postselection state $\left|\psi_{2}\right\rangle=|V \pm \Delta\rangle$ or $|H \pm \Delta\rangle$, with $\Delta \ll 1$ being the postselection angle. The two opposite spin components will undergo destructive interference at the surface of the second polarizer, which makes the amplified displacement in the meter much larger than the initial one. Remarkably, the free evolution of the light beam can also enhance the pointer shift due to the propagation amplification. We use a CCD to measure the total amplified displacement after a long-focal-length lens (L2).
Our tunneling structure is a three-layer model with the metal film embed between two BK7 right angle prisms. Firstly, we coat on the hypotenuse face of one prism with metal film. When a beam reflected on the prism coated with $\mathrm{Au}$ film, we can determine the accurate width of the tunneling potential barrier by detecting the photonic $\mathrm{SHE}^{27,28}$. The permittivity of $\mathrm{Au}$ is chosen as $\varepsilon=-10.4+1.4 i$ at $632.8 \mathrm{~nm}^{29}$. We choose the initial waist radius $w_{0} \simeq 27 \mu \mathrm{m}$ (See supplementary materials for determining the size of waist radius). With the aid of an index matching fluid, the two prepared prisms (one coated with metal film and the other not) are clamped together to build up the photon tunneling structure. Here, the metal film can be seen as a tunneling barrier because its potential is larger than zero, however, the total energy of the incident photon in BK7 prism is less than zero.

When the preselection and postselection states are orthogonal, i.e., $\Delta=0$, we can look forward to detecting the cross-polarized components, hence, to register the spin-dependent splitting ${ }^{22}$. The crosspolarized field distributions are described in Fig. 3. Here, we use the 
polarizer P1 to get the preselection state as $\left|\psi_{1}\right\rangle=|H\rangle$ or $|V\rangle$ and the second polarizer P2 to obtain the postselection state $\left|\psi_{2}\right\rangle=|V\rangle$ or $|H\rangle$, respectively. The cross components suggest that photons with opposite helicities accumulate at the opposite edges of the beam, and thereby provide a direct evidence of photonic SHE in tunneling. We note that this effect is very similar to another experiment about doing phase estimation ${ }^{30}$ and is in fact related to the inverse weak value ${ }^{31}$. By making the state overlap the smallest parameter, the mode splits into two and be used to estimate the complementary variable. It is also noted that there exists strong scattering background for preselection state $|H\rangle$ [Fig. 3(c)]. The main reason is that, under this condition, the surface plasmon resonance of metal can be excited and the corresponding reflected field changes.

We measure the amplified displacements varying with the postselection angles as shown in Fig. 4. To avoid the influence of surface quality factor of metal film, we carried out the experiments for three different areas of the tunneling sample. We prepare the metal $(\mathrm{Au})$ tunneling potential barrier with three different thicknesses 9,12 , and $16 \mathrm{~nm}$ (See supplementary materials for confirming the actual thickness of tunneling barrier). After confirming the actual thickness of the metal tunneling potential barrier, we can observe the photonic SHE in this tunneling structure. For the preselection state $|H\rangle$, the initial transverse shifts are 9.1, 14.0, and $26.4 \mathrm{~nm}$, respectively. For the preselection state $|V\rangle$, the initial transverse shifts are $-5.4,-9.1$, and $-15.1 \mathrm{~nm}$, respectively. We find that the transverse shifts are even larger than the potential barrier thickness. We also note that the weak value discussed here cannot be arbitrarily large when the overlap of preselection and postselection states is close to orthogonal. In fact, there exists the maximum output of the weak measurements, which is corresponding to the previous theoretical work ${ }^{32}$.

\section{Discussion}

Noted that the experimentally measured initial transverse shifts represent a slight deviation from the theoretical value due to the measurability of this tiny effect is limited by several technical issues. Firstly, the effective permittivity of a metal film can not be determined exactly due to the fact that its value depends on its thickness, surface roughness, and even polarization ${ }^{33}$. Secondly, the surface outof-flatness of prisms (about $\lambda / 10$ at $632.8 \mathrm{~nm}$ ) make it difficult to accurately determine the thickness of barrier. To solve this problem, we use the index matching fluid to bond the two prisms together for eliminating the surface roughness. To make the results more reliable, we carry out the comparative experiment when the two BK7 prisms are put together only using the index matching fluid (without coating the $\mathrm{Au}$ film). The experimental results clearly show that the shifts of field centroid is nearly to zero, which indicates that there is no spindependent splitting in this situation (See supplementary materials for the comparative experiment using only index matching fluid).

In conclusion, we have demonstrated the photonic SHE in tunneling via weak measurements. The question how much time does a tunneling particle spend in the potential barrier region has remained controversial since the early days of quantum theory. In fact, by considering the appropriate time operator, the weak measurements can be a potential way for determining the tunneling time $\mathrm{e}^{34,35}$. Recently, Gehring et al. ${ }^{36}$ have investigated the contribution of the Goos-Hänchen shift to tunneling time in frustrated total internal reflection. They found that the contribution of the Goos-Hänchen displacement is in fact negligible in this two-dimensional tunneling system. However, in this work, the photon tunneling effect is a threedimensional process and we find that the transverse shift is even larger than the potential barrier thickness when spin-polarized photons tunneling through a potential barrier. Therefore, it may be interesting to study the time that a tunneling particle spend in the potential barrier region by considering the photonic SHE phenomenon.

\section{Methods}

Sample preparation. Our tunneling structure is a three-layer model with the metal film embed between two BK7 right angle prisms. Firstly, we coat on the hypotenuse face of one prism with $\mathrm{Au}$ film using the electron beam evaporation technique. Then, with the aid of an index matching fluid, the two prepared prisms (one coated with $\mathrm{Au}$ film and the other not) are clamped together to build up the sample.

Experimental measurements. Experiments are conducted under room temperature inside a dark environment which is introduced to minimize the impact of external light. We measure the amplified spin-dependent shifts changing with the incident angle to determine the accurate width of the tunneling potential barrier. Then, with the known potential barrier, we observe the photonic SHE in tunneling with weak measurements. Here, the incident angle is fixed and the postselection angle varies.

1. Winful, H. Tunneling time, the Hartman effect, and superluminality: A proposed resolution of an old paradox. Phys. Rep. 436, 1 (2006).

2. Steinberg, A. M., Kwiat, P. G. \& Chiao, R. Y. Measurement of the single-photon tunneling time. Phys. Rev. Lett. 71, 708 (1993).

3. Balcou, Ph. \& Dutriaux, L. Dual optical tunneling times in frustrated total internal reflection. Phys. Rev. Lett. 78, 851 (1997).

4. Hooper, I. R., Preist, T. W. \& Sambles, J. R. Making tunnel barriers (including metals) transparent. Phys. Rev. Lett. 97, 053902 (2006).

5. Chiao, R. Y., Kwiat, P. G. \& Steinberg, A. M. Analogies between electron and photon tunneling: A proposed experiment to measure photon tunneling times. Physica B 175, 257 (1991).

6. Steinberg, A. M. \& Chiao, R. Y. Tunneling delay times in one and two dimensions. Phys. Rev. A 49, 3283 (1994).

7. Winful, H. G. \& Zhang, C. Tunneling delay time in frustrated total internal reflection. Phys. Rev. A 79, 023826 (2009).

8. Luo, H., Wen, S., Shu, W. \& Fan, D. Spin Hall effect of light in photon tunneling. Phys. Rev. A 82, 043825 (2010).

9. Fedorov, F. I. Theory of total reflection. Dokl. Akad. Nauk SSSR 105, 465 (1955).

10. Imbert, C. Calculation and experimental proof of the transverse shift induced by total internal reflection of a circularly polarized light beam. Phys. Rev. D 5, 787 (1972).

11. Aharonov, Y., Albert, D. Z. \& Vaidman, L. How the result of a measurement of a component of the spin of a spin $-1 / 2$ particle can turn out to be 100. Phys. Rev. Lett. 60, 1351 (1988).

12. Ritchie, N. W. M., Story, J. G. \& Hulet, R. G. Realization of a measurement of a "weak value" Phys. Rev. Lett. 66, 1107 (1991).

13. Pryde, G. J., O’Brien, J. L., White, A. G., Ralph, T. C. \& Wiseman, H. M. Measurement of quantumWeak values of photon polarization. Phys. Rev. Lett. 94, 220405 (2005).

14. Lorenzo, A. Di. \& Egues, J. C. Weak measurement: Effect of the detector dynamics. Phys. Rev. A 77, 042108 (2008).

15. Dressel, J., Bliokh, K. Y. \& Nori, F. Classical field approach to quantum weak measurements. Phys. Rev. Lett. 112, 110407 (2014).

16. Dixon, P. B., Starling, D. J., Jordan, A. N. \& Howell, J. C. Ultrasensitive beam deflection measurement via interferometric weak value amplification. Phys. Rev. Lett. 102, 173601 (2009).

17. Brunner, N. \& Simon, C. Measuring small longitudinal phase shifts: Weak measurements or standard interferometry? Phys. Rev. Lett. 105, 010405 (2010).

18. Lundeen, J. S., Sutherland, B., Patel, A., Stewart, C. \& Bamber, C. Direct measurement of the quantum wavefunction. Nature 474, 188-191 (2011).

19. Kocsis, S. et al. Observing the average trajectories of single photons in a Two-Slit interferometer. Science 332, 1170-1173 (2011).

20. Salvail, J. Z. et al. Full characterization of polarization states of light via direct measurement. Nature Photon. 7, 316-321 (2013).

21. Onoda, M., Murakami, S. \& Nagaosa, N. Hall effect of light. Phys. Rev. Lett. 93, 083901 (2004).

22. Bliokh, K. Y. \& Bliokh, Y. P. Conservation of angular momentum, transverse shift, and spin Hall effect in reflection and refraction of an electromagnetic wave packet. Phys. Rev. Lett. 96, 073903 (2006).

23. Hosten, O. \& Kwiat, P. Observation of the spin Hall effect of light via weak measurements. Science 319, 787-790 (2008).

24. Shitrit, N. et al. Spin-Optical metamaterial route to Spin-Controlled photonics. Science 340, 724-726 (2013).

25. Jordan, A. N., Martínez-Rincón, J. \& Howell, J. C. Technical advantages for WeakValue amplification: When less is more. Phys. Rev. X 4, 011031 (2014).

26. Dressel, J., Malik, M., Miatto, F. M., Jordan, A. N. \& Boyd, R. W. Colloquium: Understanding quantum weak values: Basics and applications. Rev. Mod. Phys. 86, 307 (2014).

27. Zhou, X., Xiao, Z., Luo, H. \& Wen, S. Experimental observation of the spin Hall effect of light on a nanometal film via weak measurements. Phys. Rev. A 85, 043809 (2012).

28. Zhou, X., Ling, X., Luo, H. \& Wen, S. Identifying graphene layers via spin Hall effect of light. Appl. Phys. Lett. 101, 251602 (2012).

29. Palik, E. D. Handbook of Optical Constants of Solids (Academic Press, New York, 1998). 
30. Starling, D. J., Dixon, P. B., Williams, N. S., Jordan, A. N. \& Howell, J. C. Continuous phase amplification with a Sagnac interferometer. Phys. Rev. A 82, 011802(R) (2010).

31. Kofman, A. G., Ashhab, S. \& Nori, F. Nonperturbative theory of weak pre- and post-selected measurements. Phys. Rep. 520, 43 (2012).

32. Lorenzo, A. Di. Weak values and weak coupling maximizing the output of weak measurements. Ann. Phys. 345, 178 (2014)

33. Barnes, W. L. Comparing experiment and theory in plasmonics. J. Opt. A: Pure Appl. Opt. 11, 114002 (2009).

34. Steinberg, A. M. How much time does a tunneling particle spend in the barrier region? Phys. Rev. Lett. 74, 2405 (1995).

35. Choi, Y. \& Jordan, A. N. Operational approach to indirectly measuring the tunneling time. Phys. Rev. A 88, 052128 (2013).

36. Gehring, G. M., Liapis, A. C., Lukishova, S. G. \& Boyd, R. W. Time-Domain measurements of reflection delay in frustrated total internal reflection. Phys. Rev. Lett. 111, 030404 (2013).

\section{Acknowledgments}

This research was supported by the National Natural Science Foundation of China (Grants No. 61025024, No. 11274106, and No. 11474089).

\section{Author contributions}

H.L. conceived the idea and designed the experiment. The experiment was performed by X.Z., X.Z., X.L. and Z.Z. contributed to the theoretical work and analyzed the data; H.L. and S.W. supervised all aspects of the project. All authors contributed to the text of the manuscript.

\section{Additional information}

Supplementary information accompanies this paper at http://www.nature.com/ scientificreports

Competing financial interests: The authors declare no competing financial interests.

How to cite this article: Zhou, X., Ling, X., Zhang, Z., Luo, H. \& Wen, S. Observation of Spin Hall Effect in Photon Tunneling via Weak Measurements. Sci. Rep. 4, 7388; DOI:10.1038/ srep07388 (2014).

This work is licensed under a Creative Commons Attribution-NonCommercialNoDerivs 4.0 International License. The images or other third party material in this article are included in the article's Creative Commons license, unless indicated otherwise in the credit line; if the material is not included under the Creative Commons license, users will need to obtain permission from the license holder in order to reproduce the material. To view a copy of this license, visit http:// creativecommons.org/licenses/by-nc-nd/4.0/ 